[Contribution from the Chemistry Section of the Iowa Agricultural EXPERIMENT STATION.]

\title{
A NOTE ON THE DUCLAUX METHOD FOR VOLATILE FATTY ACIDS.
}

By A. R. LAMB.

Received January 25, 1917.

In the course of some investigations into the fermentation of corn silage it was intended to make use of the Duclaux method ${ }^{1}$ for the determination of volatile fatty acids. A critical study of the method was therefore begun with the purpose of obtaining from it the most accurate results possible. As a result of a change in the plan of the investigation this study was discontinued about two years ago. However, in view of the fact of the publication of a criticism of the Duclaux method by Upson, Plum, and Schott ${ }^{2}$ it is considered advisable to publish a few of the results of this study of the method.

It was found that the commercial C. P. acids from various firms gave varying Duclaux constants, although repeated determinations with the same acid checked very well. Therefore formic, acetic, and propionic acids were very carefully purified by repeated crystallization of suitable salts and repeated distillation of the acids liberated from the pure salts. This precaution was apparently neglected by Upson, Plum, and Schott. The extent of these variations, which are great enough to seriously affect the analysis of a mixture, is shown in the following table:

Commercial acetic $10 \mathrm{cc} .20 \mathrm{cc} .30 \mathrm{cc} .40 \mathrm{cc} .50 \mathrm{cc} .60 \mathrm{cc} .70 \mathrm{cc} .80 \mathrm{cc} .90 \mathrm{cc} .100 \mathrm{cc}$

\begin{tabular}{|c|c|c|c|c|c|c|c|c|c|c|}
\hline$\ldots \ldots \ldots$ & $7 \cdot 3$ & 15.2 & 23.5 & 32.1 & 4 I.I & 50.6 & $6 \pi .1$ & 72.1 & 85.2 & Ic \\
\hline & 7.6 & $15 \cdot 7$ & 24.0 & 32.6 & $4 \mathrm{I} \cdot 7$ & 50.9 & $6 \mathrm{I} .4$ & 72.5 & 35.0 & 100 \\
\hline $\begin{array}{l}\text { nstants. } \\
\text { ts from } \\
\text { acetic }\end{array}$ & $7 \cdot 4$ & I5.2 & 23.4 & 32.0 & 40.9 & 50.5 & 60.9 & 71.9 & 4.4 & 100 \\
\hline & & 5.4 & 3.7 & 2.4 & 1.5 & I. I & $6 \mathrm{I} \cdot 4$ & 72.5 & 85.1 & \\
\hline
\end{tabular}

Throughout the work the experimental conditions, which were not well defined by Duclaux, were kept as nearly uniform as possible. An electric hot plate was used as the source of heat. The $200 \mathrm{cc}$. short-necked distilling flask, which was connected by a short bent tube to the condenser, was surrounded by an asbestos jacket. The ro cc. portions of the distillate were collected and measured in carefully calibrated tall form ro cc. cylinders and titrated with $0.05 \mathrm{~N}$ barium hydroxide solution. Although carbon dioxide-free water was used it was found necessary to apply to the titration figures a series of corrections for the carbon dioxide taken up during the distillation. Until electric heating was adopted under carefully regulated conditions concordant results could not be obtained. Under the above-described conditions, however, very concordant results

1 Duclaux, "Traite de Microbiologie III," 384-395 (I900).

2 ThIS JournaL, 39, 73I (I9I7). 
were obtained. Several series of about twelve distillations each, using different amounts of acid, were conducted and the Duclaux constants for the three acids mentioned above were calculated. These constants varied somewhat from Duclaux's. The differences in the case of acetic acid are shown above.

Under the same experimental conditions and using these constants, accurate results were obtained with mixtures of two acids. The following example shows the nature of the results obtained with mixtures of two acids:

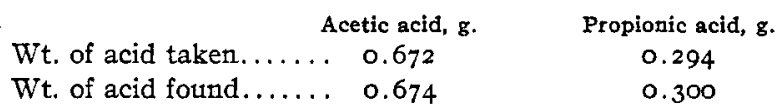

In the judgment of the writer, it is impracticable to attempt to deter mine more than two or three acids in the same fraction. By proper fractional distillation the acids in the mixture may be quite well separated. This obviates the necessity of calculating as many as four acids from a single distillation, which is objected to by Upson, et al.

It is believed by the writer that the lack of agreement between different sets of Duclaux's constants, attributed by the authors just mentioned to unavoidable experimental variations, is due instead to impurities in the acids used and to avoidable experimental variations. The results obtained by the writer under closely regulated experimental conditions, are so concordant as to warrant much closer calculation of results than has been possible in the past, thus increasing the reliability of the method. Further difficulties suggested by the authors mentioned above might have been avoided by the use of qualitative tests to determine the presence or absence of certain acids and the relative claims to acceptance of two or more possible combinations.

In view of the publication of a new method for volatile fatty acids by steam distillation ${ }^{1}$ which appears to have some advantages not possible of attainment by the Duclaux method, it seems inadvisable at present to continue further a study of the Duclaux method. However, in the opinion of the writer it is possible under proper conditions to obtain much more accurate results with the method than have generally been obtained.

AMES, IOWA.

[Contribution from the Chemical Laboratory of Presidency College.]

\section{NEUTRAL POTASSIUM PERSULFATE AS A REAGENT IN ORGANIC CHEMISTRY.}

By Rasig Ial, Datta and Jnanendra Nata Sen.

Received February 16, 1917.

The object of this investigation is to study the action of potassium persulfate on organic bodies in order to find out the many interesting

2 Dyer, J. Biol. Chem., 28, 445-473 (1917). 